\title{
Synthesis and NMR studies of novel 2-eteroarylbenzofuran derivatives
}

\author{
Giovanna Delogu ${ }^{1}$, Elias Quezada ${ }^{2}$ \\ ${ }^{1}$ Dipartimento di Scienze della Vita e dell'Ambiente, Università degli Studi di Cagliari, Via Ospedale 72, 09124 \\ Cagliari, Italy. \\ ${ }^{2}$ Departamento de Quimica Organica, Facultad de Farmacia, Universidad de Santiago de Compostela, 15782, \\ Santiago de Compostela, Spain
}

Email: giovannadelogu@ hotmail.it

\begin{abstract}
:
The benzofuran scaffold is ubiquitous in the area of pharmacologically active agents and isolated natural products. Natural products possessing the 2-substituted moiety exhibit a broad range of pharmacological activities such as antimicrobial, anti-inflammatory, antifungal, antipsychotic, antilipidemic, analgesic, cytotoxic and central nervous system stimulant. Also, benzofuran nucleus has been recently described as a good scaffold for designing potent and relatively non-selective MAOIs.

The synthesis and identification of novel 2-eteroarylbenzofuran derivatives with electrondonating and electron-withdrawing substituents are reported in this work.

The key step for the formation of the benzofuran moiety was achieved by an intramolecular Wittig reaction between ortho-hydroxybenzyltriphosphonium salts and the appropriate aroyl chlorides.

Their complete structural characterization was performed using one-dimensional and twodimensional resonance techniques. The acquired data constitute a valuable database for the unambiguous identification of eteroarylbenzofuran library developed with the aim of our medicinal chemistry program.
\end{abstract}

Keywords: synthesis, eteroarylbenzofuran, NMR

\section{Introduction:}

Many important biochemical compounds and drugs of natural origin contain heterocyclic ring structures. The presence of heterocyclic structure in such different type of compounds is strongly 
indicative of the diverse pharmacological activities and recognition of this is reflected in efforts to find useful synthetic drugs.

The benzofuran ring system itself is a common structure element that appears in a large number of medicinally important compounds. ${ }^{1}$ Widespread interest in the chemistry of benzofurans in a large number of natural products has attracted due to their biological activities and their potential application as pharmacological agents. ${ }^{2}$

Various benzofuran derivatives possess different pharmacological and biological activities of which the most potent is anti-inflammatory, pesticidal e insecticidal, antihistaminic, anticonvulsant, antiallergic. In vitro-HIV-1, anticancer, anti-microbial activities. ${ }^{3,4}$

Also, benzofuran nucleus has been recently described as a good scaffold for designing potent and relatively non-selective MAO inhibitors. ${ }^{5,6}$

In the present work a series of 2-eteroarylbenzofurans (Figure 1) were synthesized and their complete structural characterization was performed using one-dimensional and two-dimensional resonance techniques.
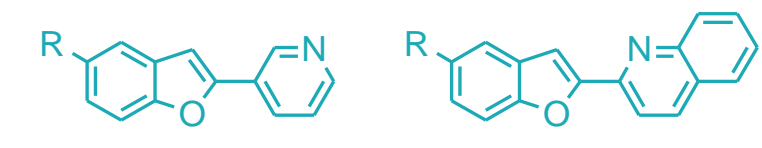

Figure 1

\section{Results and discussion}

Compounds 1-8 were efficiently synthesized according to the synthetic strategy outlined in Scheme 1. The key step for the formation of the benzofuran moiety was achieved by an intramolecular Wittig reaction between ortho-hydroxybenzyltriphosphonium salt and the appropriate aroylchloride. ${ }^{7}$

The desired Wittig reagent was readily prepared from the conveniently substituted orthohydroxybenzyl alcohol and $\mathrm{PPh}_{3} \cdot \mathrm{HBr}^{8,9}$ 


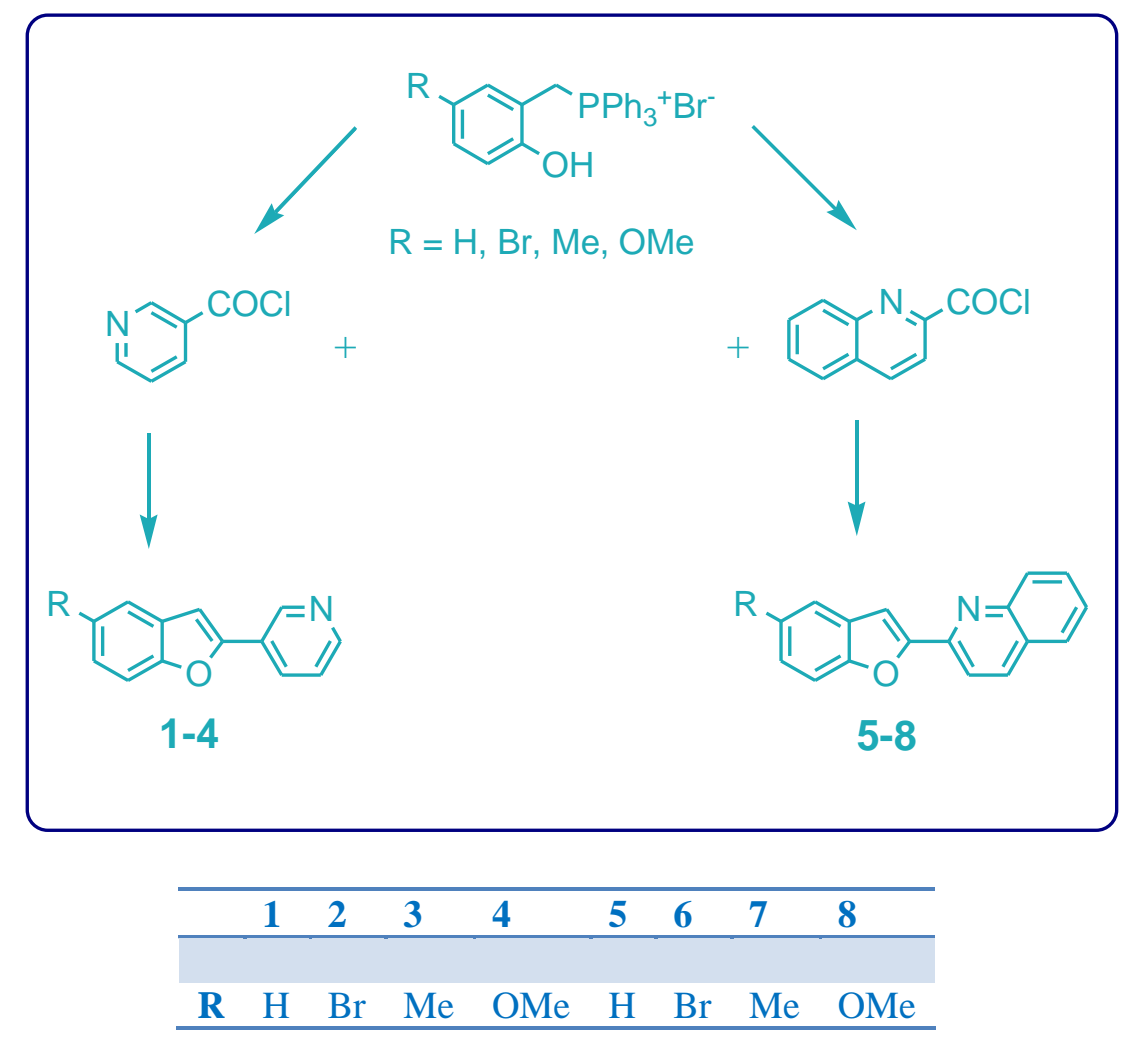

Scheme 1. Reagents and conditions: toluene, $\mathrm{NEt}_{3}, 110^{\circ} \mathrm{C}, 2 \mathrm{~h}$

The benzofurans structure were confirmed by mass spectrometry, elemental analyses, onedimensional and two- dimensional resonance techniques. In table 1 we reported yields and mp obtained for compound 1-8.

\begin{tabular}{ccc} 
compounds & Yeld & Mp \\
\hline $\mathbf{1}$ & $60 \%$ & $78-80{ }^{\circ} \mathrm{C}$ \\
\hline $\mathbf{2}$ & $17 \%$ & $109-111^{\circ} \mathrm{C}$ \\
\hline $\mathbf{3}$ & $40 \%$ & $116-118^{\circ} \mathrm{C}$ \\
\hline $\mathbf{4}$ & $10 \%$ & $129-131{ }^{\circ} \mathrm{C}$ \\
\hline $\mathbf{5}$ & $23 \%$ & $128-130{ }^{\circ} \mathrm{C}$ \\
\hline $\mathbf{6}$ & $10 \%$ & $186-188^{\circ} \mathrm{C}$ \\
\hline $\mathbf{7}$ & $15 \%$ & $154-156{ }^{\circ} \mathrm{C}$ \\
\hline $\mathbf{8}$ & $15 \%$ & $144-146{ }^{\circ} \mathrm{C}$ \\
\hline
\end{tabular}

Table 1: Yields and Mp obtained for compound 1-8 


\section{Materials and Methods}

Starting materials and reagents were obtained from commercial suppliers and were used without purification. Melting points (mp) are uncorrected and were determined with a Reichert Kofler thermopan or in capillary tubes in a Buchi 510 apparatus.

${ }^{1} \mathrm{H}$ NMR and ${ }^{13} \mathrm{C}$ NMR spectra of the samples, approximately $10 \%$ solutions in deuterated dimethyl sulfoxide, were recorded with at room temperature in 5-mm-outer-diameter tubes. TMS was used as internal standard, and chemical shifts are expressed in parts per million $(\delta)$ and $J$ in hertz. 1D ${ }^{13} \mathrm{C}$ NMR was recorded on a Bruker AMX 500 NMR spectrometer operating at 125.77 $\mathrm{MHz}$, typically with a $30^{\circ}$ pulse flip angle, a pulse repetition time of $4.8 \mathrm{~s}$, and a spectral width of $31.250 \mathrm{~Hz}$ with $32 \mathrm{~K}$ data points. For the DEPT sequence, the width of the $90 \circ$ pulse for ${ }^{13} \mathrm{C}$ was 4 $\mu \mathrm{s}$, and that of the $90^{\circ}$ pulse for ${ }^{1} \mathrm{H}$ was $9.5 \mu \mathrm{s}$; the delay $2 \mathrm{~J}_{\mathrm{C}, \mathrm{H}}$ was set to $3.5 \mathrm{~ms}$. One-bond HMQC spectra were recorded on a Bruker AMX 500 spectrometer using pulse sequence that allowed gradient selection (Bruker programs INV4GS and INVGSLPLRND). The spectra were collected in the $t_{1}$ domain in 256 experiments with $2 \mathrm{~K}$ data points and spectral widths of 5.050 and $27669 \mathrm{~Hz}$ in the $\mathrm{F}_{2}\left({ }^{1} \mathrm{H}\right)$ and $\mathrm{F} 1\left({ }^{13} \mathrm{C}\right)$ dimensions, respectively. The relaxation delay $\mathrm{D}_{1}$ was set to $2 \mathrm{~s}$. The data were processed using sine-bell weighting functions in both dimensions.

Mass spectrometry was carried out with a Hewlett-Packard 5988A spectrometer operating under EI conditions (electron energy $70 \mathrm{eV}$ ). Elemental analyses were performed using a Perkin-Elmer 240B microanalyser and were within $\pm 0.4 \%$ of calculated values in all cases. Silica gel (Merk 60, 230-00 mesh) was used for flash chromatography (FC). Analytical thin layer chromatography (TLC) was performed on plates precoated with silica gel (Merk 60 F254, 0.25 mm).

\section{Chemistry}

General procedure for the preparation of 2-hydroxybenzylalcohols: $\mathrm{NaBH}_{4}(6.60 \mathrm{mmol})$ was added to a stirring solution of 2-hy-droxybenzaldehyde $(6.60 \mathrm{mmol})$ in $\mathrm{EtOH}(20 \mathrm{~mL})$ in an ice bath. The reaction mixture was stirred at room temperature for $1 \mathrm{~h}$. After that, the solvent was removed, $1 \mathrm{n}$ aqueous $\mathrm{HCl}(40 \mathrm{~mL})$ was added to the residue and extracted with $\mathrm{Et}_{2} \mathrm{O}$. The solvent was evaporated under vacuum to give the desired compounds

General procedure for the preparation of 2-hydroxybenzyltri-phenylphosphonium bromide: A mixture of 2-hydroxybenzylalcohol $(24.6 \mathrm{mmol})$ and $\mathrm{PPh}_{3} \cdot \mathrm{HBr}(24.6 \mathrm{mmol})$ in $\mathrm{CH}_{3} \mathrm{CN}(50 \mathrm{~mL})$ was stirred under reflux for $2 \mathrm{~h}$. The solid that formed was filtered and washed with $\mathrm{CH}_{3} \mathrm{CN}$ to give 
the desired compounds.

General procedure for the preparation of 2-aroylylbenzofuran: A mixture of 2hydroxybenzyltriphenylphosphonium bromide $(1.10 \mathrm{mmol})$ and aroyl chloride $(1.11 \mathrm{mmol})$ in a mixed solvent (toluene $20 \mathrm{~mL}$ and $\mathrm{Et}_{3} \mathrm{~N} 0.5 \mathrm{~mL}$ ) was stirred under reflux for $2 \mathrm{~h}$. The precipitate was removed by filtration. The filtrate was concentrated, and the residue was purified by silica gel chromatography (hexane/EtOAc 9:1) to give the desired compounds 1-8.

\section{Conclusion}

Novel 3-(2-benzofuran-2yl)-pyridine and 2-(2-benzofuran-2-yl)-quinoline derivatives with electron-donating and electron-withdrawing substituents in 5 position of the indole ring were obtained in moderate yields by intramolecular Wittig reaction. The compounds were further characterized by $1 \mathrm{D}$ and $2 \mathrm{D}$ NMR techniques that allowed full NMR signal assignments. The acquired data constitute a valuable database for the unambiguous identification of the benzofuran library developed with the aim of our medicinal chemistry program

\section{Acknowledgements}

This work was partially supported by Università degli Studi di Cagliari and Fondazione Banco di Sardegna 2012.

\section{References}

1. S. E. Yoo, S. H. Lee, S. K. Kim, Bioorg. Med. Chem., 1997; 5, 445.

2. F. A. W. Bakr, A. A. A. Hatem, M. A. Essam, Eur. Journal of Med. Chem., 2009; 44, 2632.

3. L. Santana, M. Teijeira, E. Uriarte, C. Teran, Eur. J. Pharm. Sci., 1999, 7, 161.

4. R. Madhu, M. D. Karvekar, S. Patel, S. Sarkar, Asian J. Chem., 2009, 21, 5151.

5. $\quad$ L. H. A. Prins, J. P. Petzar, S. F. Malan, Eur. J. Med. Chem., 2010, 45, 4458.

6. G. Ferino, E. Cadoni, M. J. Matos, E. Quezada, E. Uriarte, L. Santana, M. Yanez, D. Viña, C. Picciau, S. Serra, G. Delogu, Chem. Med. Chem., 2014, 9, 1672.

7. A. Hercouet, M. Le Corre, Tetrahedron Lett., 1979, 23, 2145.

8. M. Ono, H. Kawashima, A. Nonaka, T. Kaway, M. Haratake, H. Mori, M. P. Kung, H. F. Kung, H. Saji, M. Nakayama, J. Med. Chem., 2006, 49, 2725.

9. M. Ono, M. P. Kung, C. Hou, H. F. Kung, Nucl. Med. Biol., 2002, 29, 633. 